\title{
Reliability analysis of cloud-RAID 6 with imperfect fault coverage
}

\author{
Lavanya Mandava, Liudong Xing*
}

Department of Electrical and Computer Engineering, University of Massachusetts Dartmouth, 285 Old Westport Road, Dartmouth, MA 02747, USA

\begin{abstract}
Cloud-RAID (Redundant Array of Independent Disks) is a data storage model, where different data redundancy techniques (corresponding to different levels) are utilized to enhance data reliability and availability in an anytime, anywhere data access framework implemented in the cloud environment. Such a fault-tolerant system can be subject to imperfect coverage due to imperfect fault detection or recovery mechanism, causing extensive damage to the whole system. In this paper, we model reliability of a cloud-RAID 6 storage system addressing effects of two types of imperfect coverage (element level coverage and fault level coverage). Numerical results are provided to illustrate effects of those behaviors on the system reliability performance.
\end{abstract}

Keywords: binary decision diagram; cloud-RAID; element level coverage; fault level coverage; reliability

(Submitted on February 23, 2017; Revised on April 20, 2017; Accepted on April 22, 2017)

(C) 2017 Totem Publisher, Inc. All rights reserved.

\section{Introduction}

Many companies have provided cloud storage platforms such as Dropbox, Google Drive, and Amazon EC2, which allow users to enjoy on-demand, anytime, anywhere services from a shared pool of configurable resources in the cloud [1-3]. Any system failure or interruption in the service can have negative and serious effects on the reputation of cloud service providers [4]. Therefore, it is significant to address the reliability issue for the cloud storage systems [5].

Diverse solutions have been explored for achieving reliability in the cloud storage system. For example, a game-theoretic analysis method based on replications [6] was proposed to obtain optimal reliable strategies for users and cloud storage providers in [7]. In reference [8], erasure coding was applied to enhance reliability of cloud storage. In [9] a content storage and delivery mechanism was designed to tolerate failures of cloud servers. In this work, we focus on Cloud-RAIDs (Redundant Array of Independent Disks) [5, 10, 11], which represent a common class of reliability solutions based on different data redundancy techniques [12, 13]. In [14] a hierarchical method integrating a continuous-time Markov chain and a multi-valued decision diagram was suggested for evaluating reliability of a cloud-RAID storage system with heterogeneous disks from different providers. However, the method used in [14] fails to consider an inherent behavior of the cloud-RAID system, which is imperfect fault coverage [15]. Specifically, the system's fault recovery mechanism, which is responsible for fault detection, fault location, fault isolation and system reconfiguration, can seldom be fully reliable. When the mechanism fails, the undetected fault may propagate, causing extensive damages to the overall system.

In this paper, we present combinatorial methods to address effects of imperfect fault coverage in the reliability analysis of a cloud-RAID 6 system, one level of the cloud-RAIDs [16] described in Section 2. Two types of imperfect coverage models including element level coverage (ELC) and fault level coverage (FLC) (Section 3) are considered.

* Corresponding author. Tel.: +1-508-999-8883; Fax: +1-508-999-8489.

E-mail address: 1xing@umassd.edu. 


\section{Cloud-RAID 6 system}

In a cloud-RAID 6 system, data to be stored are separated into blocks, which are then striped across multiple disks within the array [17]. Double parity is implemented, where the parity stripes are also distributed across the multiple disks. Figure 1 illustrates the structure of an example cloud-RAID 6 system with five disk drives that may come from different cloud providers. Blocks located on the same row of the array form a virtual disk drive with two of them storing the parity information for achieving fault tolerance. Specifically, the system can tolerate malfunctions of any two disk drives; the malfunctioned stripes can be restored using the remaining data stripes and parity stripes. Thus, the example cloud-RAID 6 system can be modeled using a 3-out-of-5: G model, where the whole system is considered being reliable if at least three disks are operating correctly.

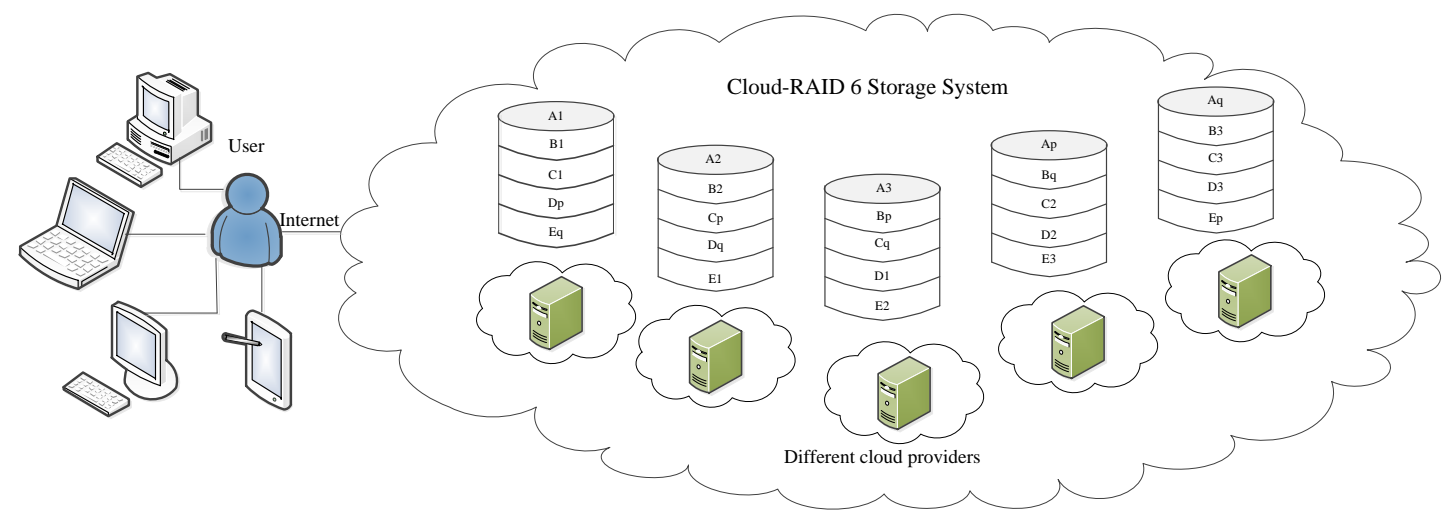

Figure 1. Architecture of an example cloud-RAID 6

\section{Preliminary models}

This section presents basics of the ELC and FLC models and binary decision diagrams used in the combinatorial methods for the reliability analysis of the cloud-RAID 6 system.

\subsection{Imperfect fault coverage modeling}

Different types of models representing the imperfect fault coverage behavior are available [15, 18]. This work focused on two types of them: ELC (or single-fault model) and FLC (or multi-fault model). Under the ELC, the fault coverage probability or factor is associated with a particular element, which is independent of statuses of other elements within the same system. A system with multiple elements can tolerate multiple co-existing single element faults. Under the FLC, the fault coverage probability relies on the number of element faults happening to a particular group within a certain recovery window. The ELC model is appropriate for systems with elements having self-diagnostic capability. The FLC model is often applied to multiprocessor systems in load-sharing environments [19] or control systems in aircrafts applications [20, 21].

\subsection{Binary decision diagram $(B D D)$}

A BDD is a rooted, directed acyclic graph based on Shannon decomposition rule of $f=x f_{x=1}+\bar{x} f_{x=0}=x F_{1}+\bar{x} F_{0}$, where $f$ is a Boolean function on a set of Boolean variables including $x[15,22]$. The rule can also be compactly represented using the ite (if-then-else) format as shown in (1).

$$
f=\operatorname{ite}\left(x, F_{1}, F_{0}\right)
$$

Figure 2 illustrated the rule graphically, where $x$ corresponds to a non-sink node in the BDD model with two outgoing edges: ' 1 '-edge/then-edge (denoted by dashed line) leading to child node $F_{1}$ and ' 0 '-edge/else-edge (denoted by solid line) leading to child node $F_{0}[22,23]$. The then and else edges are associated with probabilities $q(x)$ (component unreliability) and $p(x)$ (component reliability), respectively.

A system BDD model has two sink nodes '1' and '0', representing system failure and operation, respectively. Equation (2) 
presents rules for combining two sub-BDD models represented by $g$ and $h$ into one BDD model, which are essentially manipulation rules for generating a system BDD model [18].

$$
\begin{aligned}
g \diamond h= & \operatorname{ite}\left(x, G_{1}, G_{0}\right) \diamond \operatorname{ite}\left(y, H_{1}, H_{0}\right) \\
= & \begin{cases}\text { ite }\left(x, G_{1} \diamond H_{1}, G_{0} \diamond H_{0}\right) & \text { index }(x)=\operatorname{index}(y) \\
\text { ite }\left(x, G_{1} \diamond h, G_{0} \diamond h\right) & \text { index }(x)<\operatorname{index}(y) \\
\text { ite }\left(y, g \diamond H_{1}, g \diamond H_{0}\right) & \text { index }(x)>\operatorname{index}(y)\end{cases}
\end{aligned}
$$

For the BDD generation, each Boolean variable modeling a system component is assigned an index representing the order of the variable in the input ordering list. During the generation or combination, indices of two root nodes ( $x$ for $g$ and $y$ for $h$ ) are compared. In the case of $x$ and $y$ having the same index, the logic operation denoted by $\diamond$ is applied to their child nodes as shown in the first rule of (2). Otherwise, the node having a smaller index becomes the root node of the combined model and the logic operation is applied to each child node of the smaller-index node, and the larger-index node. With the system BDD model generated, the system unreliability can be computed by adding probabilities of all paths from root to sink node ' 1 '.

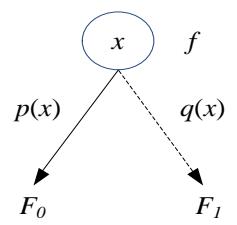

Figure 2. A non-sink node in the BDD model encoding an ite format

\section{Reliability of cloud-RAID 6 with ELC}

In this section, we present a combinatorial method based on the simple and efficient algorithm [24, 25] for evaluating reliability of the cloud-RAID 6 system considering ELC.

\subsection{Evaluation method}

Figure 3 illustrates the ELC model [24], where the entry point denotes an element fault occurring. In response to this element fault, there are three possible outcomes modeled by three exits: $R$ exit (transient restoration; the fault is transient and can be recovered without changing system status), $C$ exit (permanent coverage; the fault is permanent and the faulty element is isolated or removed), and $S$ exit (single-point failure; the fault is uncovered and propagates causing failure of the entire system). Occurrence probabilities of the three exits are denoted by $r, c, s$, and they sum to one.

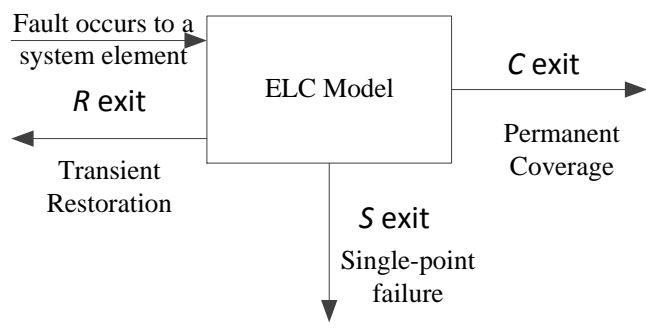

Figure 3. Structure of the ELC model [24]

Consider a single disk $d$ in the cloud-RAID 6 system $(d=1,2,3,4,5)$. Under the ELC model, Equation (3) gives the probability that disk $d$ is functioning $(n[d])$, failed covered $(c[d])$, and failed uncovered $(u[d])$.

$$
n[d]=1-q_{d}(t)+q_{d}(t) * r_{d}, \quad c[d]=q_{d}(t) * c_{d}, \quad u[d]=q_{d}(t) * s_{d}
$$

where $q_{d}(t)$ is the fault occurrence probability of disk $d$ at time $t$.

Consider the following three events for the considered cloud-RAID 6 system. $E_{1}$ : no disks undergo a single-point of failure; $E_{2}$ : at least one disk undergoes a single-point of failure; and $E$ : the cloud-RAID 6 system fails. According to the total probability law, the cloud-RAID 6 system unreliability is $U R_{E L C}=\operatorname{Pr}(E)=\operatorname{Pr}\left(E \mid E_{1}\right) * \operatorname{Pr}\left(E_{1}\right)+\operatorname{Pr}\left(E \mid E_{2}\right) * \operatorname{Pr}\left(E_{2}\right) \cdot \operatorname{Since} \operatorname{Pr}\left(E_{1}\right)+$ 
$\operatorname{Pr}\left(E_{2}\right)=1$ and $\operatorname{Pr}\left(E \mid E_{2}\right)=1$, we have

$$
U R_{E L C}=1-\operatorname{Pr}\left(E_{1}\right)+\operatorname{Pr}\left(E \mid E_{1}\right) * \operatorname{Pr}\left(E_{1}\right)
$$

where $\operatorname{Pr}\left(E_{1}\right)=(1-u[1])(1-u[2])(1-u[3])(1-u[4])(1-u[5])$ by definition. $\operatorname{Pr}\left(E \mid E_{1}\right)$ in (4) can be evaluated using the BDD method (Section 3.2) and a modified conditional failure probability for each disk $q_{d}^{\tilde{d}}=c[d] /(1-u[d])$. Figure 4 shows the BDD model exhibiting a well-defined 3-out-of-5 lattice structure. Similar to Figure 2, the solid edge means a working disk and the dashed edge means a failed disk. Sink nodes ' 1 ' and ' 0 ' correspond to the failure and operation of the example cloud-RAID 6 system, respectively.

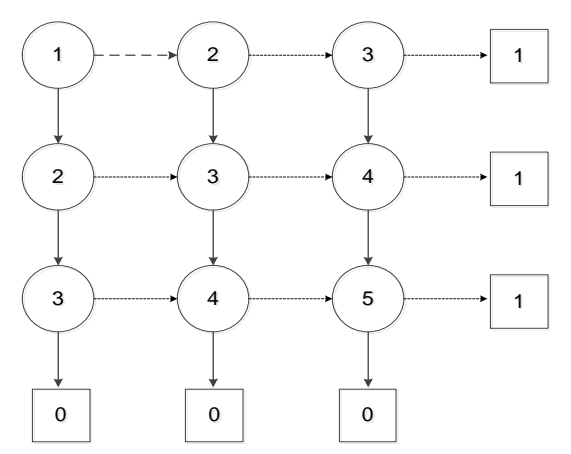

Figure 4. BDD of the example cloud-RAID 6 with ELC

Using the modified failure probabilities, $\operatorname{Pr}\left(E \mid E_{1}\right)$ can be evaluated as the sum of probabilities of all disjoint paths in Figure 4 from the root node to sink node ' 1 '.

$$
\begin{aligned}
& \operatorname{Pr}\left(E \mid E_{1}\right)=q_{1}^{\tilde{q}} \tilde{q_{2}} \tilde{q_{3}}+q_{1}^{\tilde{q}} \tilde{q_{2}}\left(1-q_{3}^{\tilde{3}}\right) \tilde{q_{4}}+q_{1}^{\tilde{q}} \tilde{q_{2}}\left(1-q_{3}^{\tilde{3}}\right)\left(1-q_{4}\right) q_{5}^{\tilde{5}} \\
& +q_{1}^{\tilde{1}}\left(1-q_{2}^{\tilde{2}}\right) \tilde{q_{3}} \tilde{q_{4}}+q_{1}^{\tilde{1}}\left(1-q_{2}^{\tilde{2}}\right)\left(1-q_{3}^{\tilde{3}}\right) \tilde{q_{4}} \tilde{q_{5}} \tilde{}+\left(1-q_{1}\right) \tilde{q_{2}} \tilde{q_{3}} \tilde{q_{4}} \tilde{} \\
& +\left(1-q_{1}^{\tilde{1}}\right)\left(1-q_{2}^{\tilde{2}}\right) \tilde{q_{3}} \tilde{q_{4}} \tilde{q_{5}} \tilde{+}+\left(1-q_{1}^{\tilde{1}}\right) q_{2}^{\tilde{(}}\left(1-q_{3}^{\tilde{3}}\right) q_{4}^{\tilde{4}} \tilde{q_{5}} \\
& \left.+\left(1-q_{1}^{\tilde{1}}\right) q_{2}^{\tilde{q_{3}}} \tilde{\left(1-q_{4}\right.}\right) q_{5}^{\tilde{5}}+q_{1}^{\tilde{1}}\left(1-q_{2}^{\tilde{2}}\right) q_{3}^{\tilde{3}}\left(1-q_{4}\right) q_{5}^{\tilde{5}}
\end{aligned}
$$

\subsection{Evaluation results}

Table 1 lists different combinations of $r, c$ and $s$ parameters for the five disks and the corresponding cloud-RAID 6 reliability $R_{E L C}=1-U R_{E L C}$ evaluated using the method of Section 4.1 for $t=1000 \mathrm{hrs}$. These results are obtained by assuming the five identical disks follow the exponential distribution with rate $\lambda_{d}=0.0001 / \mathrm{hr}\left(\right.$ i.e., $\left.q_{d}(t)=1-e^{-\lambda} d^{* t}\right)$.

Table 1 covers several special cases: 1 ) $r=1$ (meaning that all disk faults are transient), based on (3), each single disk has reliability 1 , and thus the entire system has reliability 1.2) $c=1$ (meaning that the system has perfect fault coverage), the system reliability is the same as the system without considering ELC. 3) $s=1$ (meaning any single disk fault fails the entire system), the system is essentially a series system with the worst system reliability. Cases 10,11 and 12 also correspond to perfect fault coverage, where $r+c=1$ and the system reliability increases with $r$.

Table 1. Cloud-RAID 6 reliability results considering ELC (identical disks)

\begin{tabular}{|l|c|c|c|c|l|l|l|l|c|}
\hline No. & $c$ & $r$ & $s$ & $R_{E L C}$ & No. & $c$ & $r$ & $s$ & $R_{E L C}$ \\
\hline 1 & 0 & 1 & 0 & 1 & 10 & 0.7 & 0.3 & 0 & 0.997331 \\
\hline 2 & 1 & 0 & 0 & 0.992565 & 11 & 0.5 & 0.5 & 0 & 0.998998 \\
\hline 3 & 0 & 0 & 1 & 0.606531 & 12 & 0.3 & 0.7 & 0 & 0.999777 \\
\hline 4 & 0 & 0.7 & 0.3 & 0.865177 & 13 & 0.5 & 0.3 & 0.2 & 0.907429 \\
\hline 5 & 0 & 0.5 & 0.5 & 0.783681 & 14 & 0.5 & 0.2 & 0.3 & 0.864234 \\
\hline 6 & 0 & 0.3 & 0.7 & 0.708446 & 15 & 0.3 & 0.5 & 0.2 & 0.908177 \\
\hline 7 & 0.7 & 0 & 0.3 & 0.862666 & 16 & 0.3 & 0.2 & 0.5 & 0.783479 \\
\hline 8 & 0.5 & 0 & 0.5 & 0.782776 & 17 & 0.2 & 0.5 & 0.3 & 0.865114 \\
\hline 9 & 0.3 & 0 & 0.7 & 0.708253 & 18 & 0.2 & 0.3 & 0.5 & 0.783621 \\
\hline
\end{tabular}


Table 2 presents cloud-RAID 6 reliabilities evaluated for different mission time $t$ (in hours) under three different combinations of coverage parameters. It is intuitive that the system reliability decreases with time. Comparing the second and third columns of Table 2 (sharing the same $s$ ), we observe that the system is more reliable when factor $r$ is larger. As factor $s$ increases (last column), each single disk experiences more uncovered faults, the entire system becomes less reliable.

Table 2. Cloud-RAID 6 reliability for different mission times (identical disks)

\begin{tabular}{|l|c|c|c|}
\hline$t$ & $R(c=0.5, r=0.3, s=0.2)$ & $R(c=0.3, r=0.5, s=0.2)$ & $R(c=0.2, r=0.3, s=0.5)$ \\
\hline 1000 & 0.907429 & 0.908177 & 0.783621 \\
\hline 3000 & 0.750558 & 0.762608 & 0.498631 \\
\hline 5000 & 0.618075 & 0.652358 & 0.331722 \\
\hline 10000 & 0.379642 & 0.472180 & 0.142629 \\
\hline
\end{tabular}

The evaluation method of Section 4.1 is applicable to non-identical disks. Assume the five disks have different failure rates of $\lambda_{1}=0.0001 / \mathrm{hr}, \lambda_{2}=0.0002 / \mathrm{hr}, \lambda_{3}=0.0001 / \mathrm{hr}, \lambda_{4}=0.00025 / \mathrm{hr}$ and $\lambda_{5}=0.0005 / \mathrm{hr}$. For the same combinations of $r, c$ and $s$ as in Table 1, Table 3 lists the reliability of the heterogeneous cloud-RAID 6 system considering effects of ELC, where the same observations as in the case of identical disks can be made.

Table 3. Cloud-RAID 6 reliability results considering ELC (non-identical disks)

\begin{tabular}{|l|c|c|c|c|c|c|c|c|c|}
\hline No. & $c$ & $r$ & $s$ & $R_{E L C}$ & No. & $c$ & $r$ & $s$ & $R_{E L C}$ \\
\hline 1 & 0 & 1 & 0 & 1 & 10 & 0.7 & 0.3 & 0 & 0.982474 \\
\hline 2 & 1 & 0 & 0 & 0.952787 & 11 & 0.5 & 0.5 & 0 & 0.993279 \\
\hline 3 & 0 & 0 & 1 & 0.316637 & 12 & 0.3 & 0.7 & 0 & 0.998474 \\
\hline 4 & 0 & 0.7 & 0.3 & 0.734829 & 13 & 0.5 & 0.3 & 0.2 & 0.810354 \\
\hline 5 & 0 & 0.5 & 0.5 & 0.589319 & 14 & 0.5 & 0.2 & 0.3 & 0.728771 \\
\hline 6 & 0 & 0.3 & 0.7 & 0.465814 & 15 & 0.3 & 0.5 & 0.2 & 0.815202 \\
\hline 7 & 0.7 & 0 & 0.3 & 0.719080 & 16 & 0.3 & 0.2 & 0.5 & 0.588034 \\
\hline 8 & 0.5 & 0 & 0.5 & 0.583686 & 17 & 0.2 & 0.5 & 0.3 & 0.734409 \\
\hline 9 & 0.3 & 0 & 0.7 & 0.464620 & 18 & 0.2 & 0.3 & 0.5 & 0.588928 \\
\hline
\end{tabular}

Table 4 shows the cloud-RAID 6 reliability at $t=1000 \mathrm{hrs}$ for different failure rates of disk 1 while using the same $\lambda_{2}=0.0002 / \mathrm{hr}, \lambda_{3}=0.0001 / \mathrm{hr}, \lambda_{4}=0.00025 / \mathrm{hr}, \lambda_{5}=0.0005 / \mathrm{hr}$. Apparently the system reliability declines as the failure rate of disk 1 increases.

Table 4. Cloud-RAID 6 reliability for different failure rates of disk1

\begin{tabular}{|l|c|c|c|}
\hline$\lambda_{1}$ & $R(c=0.5, r=0.3, s=0.2)$ & $R(c=0.3, r=0.5, s=0.2)$ & $R(c=0.2, r=0.3, s=0.5)$ \\
\hline 0.0001 & 0.810354 & 0.815202 & 0.588928 \\
\hline 0.0005 & 0.753245 & 0.763779 & 0.496183 \\
\hline 0.001 & 0.707557 & 0.722640 & 0.421986 \\
\hline 0.01 & 0.637138 & 0.659232 & 0.307625 \\
\hline 0.1 & 0.637129 & 0.659224 & 0.307610 \\
\hline
\end{tabular}

\section{Reliability of cloud-RAID 6 with FLC}

In this section, we present a combinatorial method for evaluating reliability of the cloud-RAID 6 systems considering effects of FLC.

\subsection{Evaluation method}

In the FLC model, a set of fault coverage factors $c_{i}$ is evaluated for a specific element group. For a $k$-out-of- $n$ : G system, factors $c_{1}, c_{2}, \ldots c_{n-k}$ are needed while $c_{i}$ for $i>(n-k)$ are considered as zero since the system fails after $(n-k+1)$ elements have failed [23]. By definition, $c_{0}$ is always 1. The evaluation of $c_{i}$ is dependent on the set of disks that have already failed. According to [19], formula (6) evaluates $c_{i}$ for systems with $n$ identical elements following the same exponential time-tofailure distribution with rate $\lambda$. The index $i$ denotes the fault number, $\tau$ denotes the fault recovery window time. This method can be easily extended to systems with non-identical elements. 


$$
c_{i}=e^{-(n-i) \lambda \tau}
$$

As mentioned in Section 2, the example cloud-RAID 6 system can be modeled as a 3-out-of-5: G system meaning that the system is functioning when at least three disks are working correctly. Thus, $c_{l}$ and $c_{2}$ are used, meaning the first disk failure is covered with $c_{1}$ and the second disk failure is covered with $c_{2}$. To consider effects of FLC, the BDD method of Section 3.2 can be applied with the insertion of corresponding coverage factor $c_{i}$ onto the relevant paths in the system BDD model.

Figure 5 illustrates the BDD model for the example cloud-RAID 6 system. To save space, only paths to sink node ' 0 ' are shown. Coverage factors $c_{0}=1, c_{1}$ and $c_{2}$ are respectively inserted to the corresponding paths involving no disk failures, 1 disk failure, and 2 disk failures.

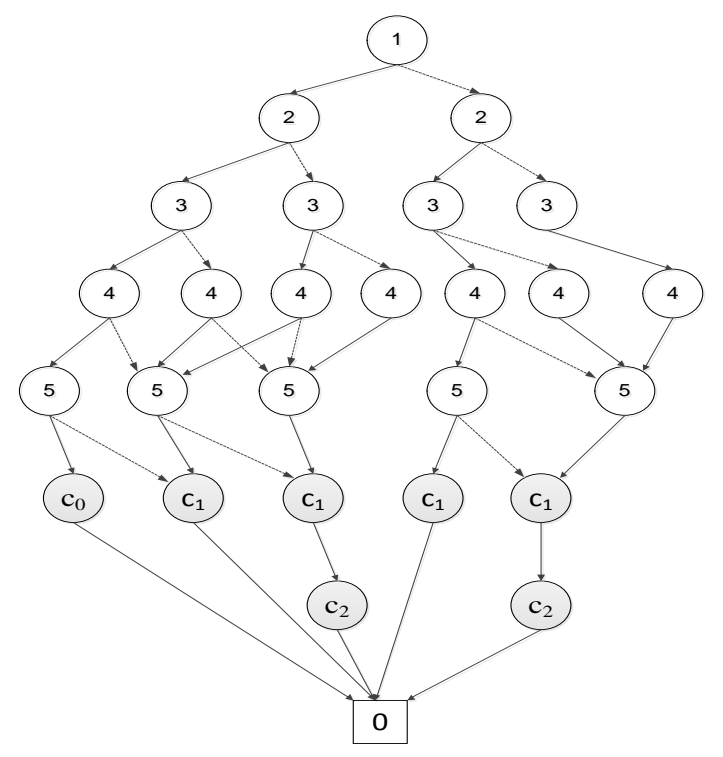

Figure 5. BDD of the example cloud-RAID 6 with FLC (identical disks)

Based on the generated BDD model in Figure 5, the reliability of the example cloud-RAID 6 system with identical disks can be evaluated by adding probabilities of all disjoint paths from the root to sink node ' 0 ' as

$$
\begin{aligned}
& R_{F L C}=p_{1} p_{2} p_{3} p_{4} p_{5} c_{0}+p_{1} p_{2} p_{3} p_{4}\left(1-p_{5}\right) c_{1}+p_{1} p_{2} p_{3}\left(1-p_{4}\right) p_{5} c_{1} \\
& +p_{1} p_{2} p_{3}\left(1-p_{4}\right)\left(1-p_{5}\right) c_{1} c_{2}+p_{1} p_{2}\left(1-p_{3}\right) p_{4} p_{5} c_{1}+p_{1} p_{2}\left(1-p_{3}\right) p_{4}\left(1-p_{5}\right) c_{1} c_{2} \\
& +p_{1} p_{2}\left(1-p_{3}\right)\left(1-p_{4}\right) p_{5} c_{1} c_{2}+p_{1}\left(1-p_{2}\right) p_{3} p_{4} p_{5} c_{1}+p_{1}\left(1-p_{2}\right) p_{3} p_{4}\left(1-p_{5}\right) c_{1} c_{2} \\
& +p_{1}\left(1-p_{2}\right) p_{3}\left(1-p_{4}\right) p_{5} c_{1} c_{2}+p_{1}\left(1-p_{2}\right)\left(1-p_{3}\right) p_{4} p_{5} c_{1} c_{2}+\left(1-p_{1}\right) p_{2} p_{3} p_{4} p_{5} c_{1} \\
& +\left(1-p_{1}\right) p_{2} p_{3} p_{4}\left(1-p_{5}\right) c_{1} c_{2}+\left(1-p_{1}\right) p_{2} p_{3}\left(1-p_{4}\right) p_{5} c_{1} c_{2} \\
& +\left(1-p_{1}\right) p_{2}\left(1-p_{3}\right) p_{4} p_{5} c_{1} c_{2}+\left(1-p_{1}\right)\left(1-p_{2}\right) p_{3} p_{4} p_{5} c_{1} c_{2}
\end{aligned}
$$

In the case of non-identical disks with different failure rates (in general, failure time distributions) or recovery window time, formula (6) needs to be modified to consider a different reliability evaluation for each disk based on its time-to-failure distribution function. The BDD in Figure 5 should also be expanded to associate a different coverage factor for paths involving the same number, but different subsets of disks failures.

Specifically, let $c_{i, d}$ denote the coverage probability associated with the $i$-th failure caused by disk $d$ in the cloud-RAID 6 $(i=1,2 ; d=1,2,3,4,5)$. The first disk failure is covered with the coverage probability $c_{1, d}$ and the second failure is covered with $c_{2, d}$. For example, if disk 2 and disk 3 fail in sequence, then the coverage probabilities of $c_{1,2}$ and $c_{2,3}$ are evaluated by extending formula (6) as

$$
c_{1,2}=e^{-\left(\lambda_{1}+\lambda_{3}+\lambda_{4}+\lambda_{5}\right) \tau_{2}}, \quad c_{2,3}=e^{-\left(\lambda_{1}+\lambda_{4}+\lambda_{5}\right) \tau_{3}}
$$

Figure 6 shows the BDD model of the example cloud-RAID 6 system with non-identical disks. Again, only paths leading to system success ( $\operatorname{sink}$ node ' 0 ') are shown. The coverage factors are inserted into the related paths. For example, in the path 
where only disk 5 fails, the coverage probability $c_{1,5}$ is inserted; in the path where disks 4 and 5 fail, coverage probabilities $c_{1,4}, c_{2,5}$ are inserted.

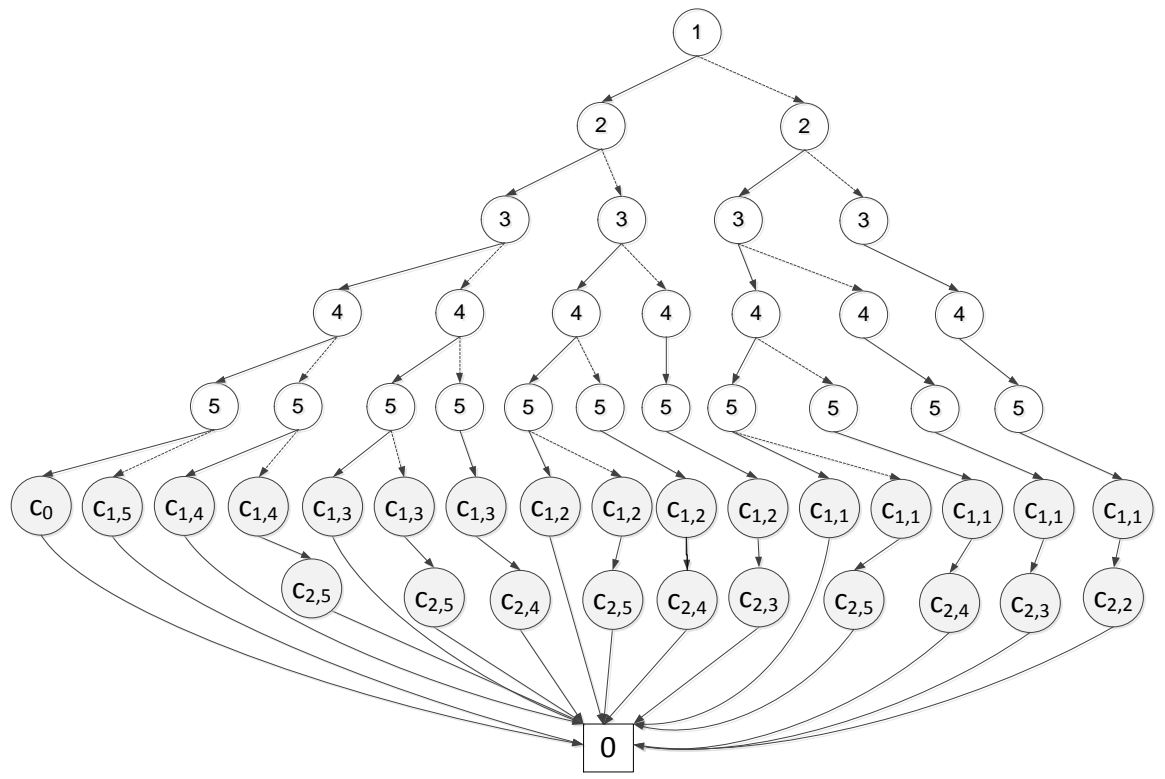

Figure 6. BDD of the example cloud-RAID 6 with FLC (non-identical disks)

Based on the generated BDD in Figure 6, the reliability of the heterogeneous cloud-RAID 6 system with FLC can be evaluated by adding probabilities of all disjoint paths from root to sink node ' 0 ' as

$$
\begin{aligned}
& R_{F L C}=p_{1} p_{2} p_{3} p_{4} p_{5} c_{0}+p_{1} p_{2} p_{3} p_{4}\left(1-p_{5}\right) c_{1,5}+p_{1} p_{2} p_{3}\left(1-p_{4}\right) p_{5} c_{1,4} \\
& +p_{1} p_{2} p_{3}\left(1-p_{4}\right)\left(1-p_{5}\right) c_{1,4} c_{2,5}+p_{1} p_{2}\left(1-p_{3}\right) p_{4} p_{5} c_{1,3}+p_{1} p_{2}\left(1-p_{3}\right) p_{4}\left(1-p_{5}\right) c_{1,3} c_{2,5} \\
& +p_{1} p_{2}\left(1-p_{3}\right)\left(1-p_{4}\right) p_{5} c_{1,3} c_{2,4}+p_{1}\left(1-p_{2}\right) p_{3} p_{4} p_{5} c_{1,2}+p_{1}\left(1-p_{2}\right) p_{3} p_{4}\left(1-p_{5}\right) c_{1,2} c_{2,5} \\
& +p_{1}\left(1-p_{2}\right) p_{3}\left(1-p_{4}\right) p_{5} c_{1,2} c_{2,4}+p_{1}\left(1-p_{2}\right)\left(1-p_{3}\right) p_{4} p_{5} c_{1,2} c_{2,3}+\left(1-p_{1}\right) p_{2} p_{3} p_{4} p_{5} c_{1,1} \\
& +\left(1-p_{1}\right) p_{2} p_{3} p_{4}\left(1-p_{5}\right) c_{1,1} c_{2,5}+\left(1-p_{1}\right) p_{2} p_{3}\left(1-p_{4}\right) p_{5} c_{1,1} c_{2,4} \\
& +\left(1-p_{1}\right) p_{2}\left(1-p_{3}\right) p_{4} p_{5} c_{1,1} c_{2,3}+\left(1-p_{1}\right)\left(1-p_{2}\right) p_{3} p_{4} p_{5} c_{1,1} c_{2,2}
\end{aligned}
$$

\subsection{Evaluation results}

Assuming all the five disks follow the same exponential distribution with rate of $0.0001 / \mathrm{hr}$. Table 5 lists values of coverage factors $c_{1}$ and $c_{2}$ calculated using (6) for different recover window time $\tau$ (in hours) and corresponding cloud-RAID 6 reliability evaluated for $t=1000 \mathrm{hrs}$ using (7). Figure 7 plots the coverage probabilities and the system reliability trend as the recovery window time $\tau$ increases.

In Table 5 when $c_{1}$ and $c_{2}$ are 1 , the system reliability is the highest, which is actually equal to the reliability of the cloud RAID 6 system with perfect fault coverage (Table 1, case No. 2). As the coverage probabilities decrease, the system reliability gets worse.

Table 5. Reliability of cloud-RAID 6 with FLC (identical disks)
\begin{tabular}{|l|c|c|c|}
\hline$\tau$ & $c_{1}$ & $c_{2}$ & $R_{F L C}$ \\
\hline 0 & 1 & 1 & 0.992565 \\
\hline 1000 & 0.670320 & 0.740818 & 0.853642 \\
\hline 2000 & 0.449329 & 0.548812 & 0.766386 \\
\hline 3000 & 0.301194 & 0.406569 & 0.710811 \\
\hline 5000 & 0.135335 & 0.223130 & 0.651721 \\
\hline 10000 & 0.018316 & 0.049787 & 0.612433 \\
\hline 15000 & 0.002479 & 0.011109 & 0.607323 \\
\hline 20000 & 0.000335 & 0.002479 & 0.606638 \\
\hline
\end{tabular}


In the case of non-identical disks, the reliability of the example cloud-RAID 6 system with FLC is calculated from (9). Assume $\lambda_{1}=0.0001 / \mathrm{hr}, \lambda_{2}=0.0002 / \mathrm{hr}, \lambda_{3}=0.0001 / \mathrm{hr}, \lambda_{4}=0.00025 / \mathrm{hr}, \lambda_{5}=0.0005 / \mathrm{hr}$ and $\tau$ to be identical for all the disks. At mission time $t=1000 \mathrm{hrs}$, Table 6 lists the system reliability for different values of $\tau$ and for different mission time $t$ with $\tau=5$ hrs. Figure 8 illustrates the graphical representation of results in Table 6 . It can be observed that the system is more reliable when the fault recovery window time is lower and the system reliability declines with the mission time.

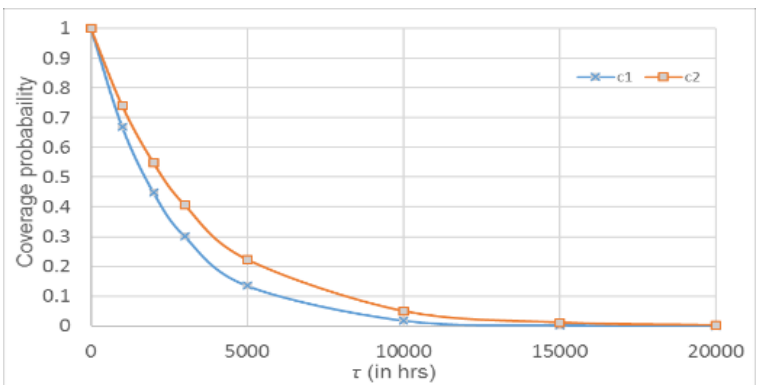

(a) Coverage probabilities

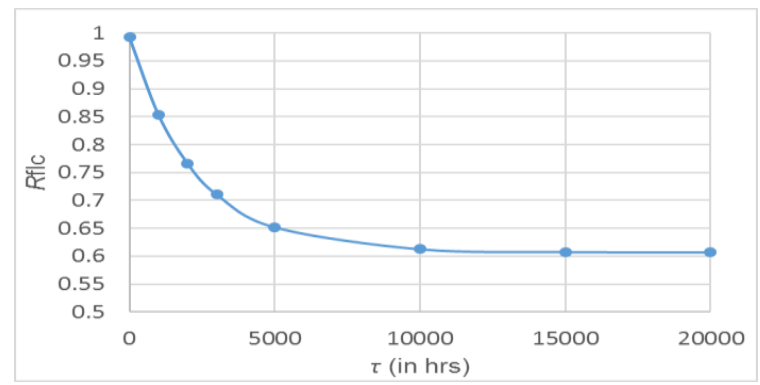

(b) System reliability

Figure 7. Cloud-RAID 6 with FLC (identical disks)

Table 6. System reliability for different $\tau$ and different $t$

\begin{tabular}{|l|c|l|c|}
\hline$\tau$ & $R_{F L C}$ & $t$ & $R_{F L C}$ \\
\hline 0 & 0.95278668 & 1000 & 0.949487567 \\
\hline 10 & 0.946207782 & 1100 & 0.937440149 \\
\hline 20 & 0.939705686 & 1200 & 0.924215323 \\
\hline 30 & 0.933279394 & 1300 & 0.909902954 \\
\hline 50 & 0.920650298 & 1500 & 0.878404804 \\
\hline 100 & 0.890336907 & 1700 & 0.843747102 \\
\hline 200 & 0.834728295 & 2000 & 0.787565065 \\
\hline
\end{tabular}

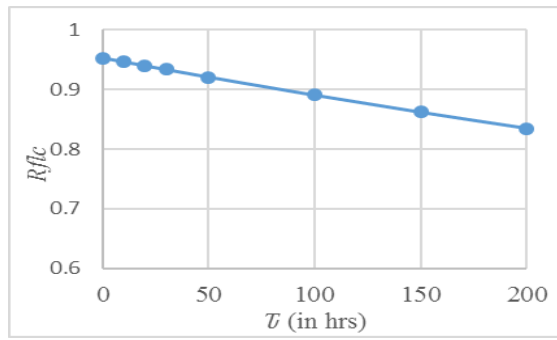

(a) Effect of $\tau$

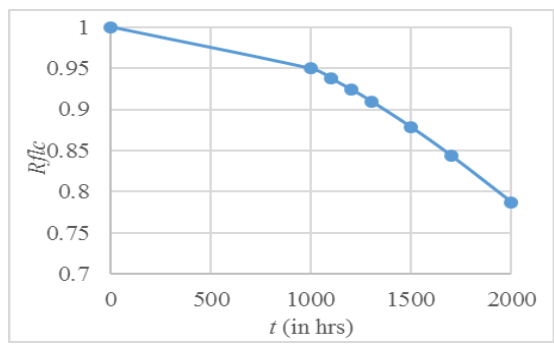

(b) Effect of $t$

Figure 8. Cloud-RAID 6 with FLC (non-identical disks)

\section{Conclusions}

Existing works on the cloud-RAID system reliability have typically assumed fully reliable fault detection and recovery mechanisms, i.e., perfect fault coverage, which is rarely true in practice. This paper relaxes this assumption through BDDbased combinatorial approaches for the reliability analysis of the cloud-RAID 6 system based on double-bit parity code. Effects of both element and fault level coverage are addressed. The methods are applicable to homogenous or heterogeneous disks. While the illustrative examples use exponential time-to-failure distributions for disks, the presented methods are applicable to arbitrary types of distributions. Numerical results demonstrate that the imperfect fault coverage can affect accuracy of the system reliability results significantly. Therefore, it is significant to consider its effect for effectively guiding system design, operations management, and optimization activities.

\section{References}

1. T. Erl, R. Puttini, and Z. Mahmood, "Cloud Computing Concepts, Technology \& Architecture," The Prentice Hall Service Technology Series, Prentice Hall, 2013 
2. J. Deng, S. C. H. Huang, Y. S. Han, and J. H. Deng, "Fault Tolerant and Reliable Computation in Cloud Computing," Proc. of IEEE Globecom workshops, pp. 1601-1605, December 2010

3. C. Wang, L. Xing, H. Wang, Y. Dai, and Z. Zhang, "Performance Analysis of Media Cloud-Based Multimedia Systems with Retrying Fault-Tolerance Technique," IEEE Systems Journal, Special Issue on Recent Advances in Cloud-based Multimedia Systems, vol. 8, no. 1, pp. 313-321, 2014

4. G. Robinson, A. Narin, and C. Elleman, "Using Amazon Web Services for Disaster Recovery," Amazon web services, 2013

5. R. Zhang, C. Lin, K. Meng, and L. Zhu, "A modeling reliability analysis technique for cloud storage system," Proc. of 15th IEEE Intl. Conf. on Communication Technology, pp. 32-36, November 2013

6. W. Li, Y. Yang, and J. Chen, "A cost-effective mechanism for cloud data reliability management based on proactive replica checking," IEEE/ACM International Symposium on Cluster, Cloud and Grid Computing, pp. 564-571, May 2012

7. C. Y. Lin, and W. G. Tzeng, "Game-Theoretic Strategy Analysis for Data Reliability Management in Cloud Storage Systems," Intl. Conf. on Software Security and Reliability, pp. 187-195, July 2014

8. Y. Lu, and S. Xia, "Novel erasure codes with repair optimality for cloud storage," International Conference on Information and Communications Technologies, pp. 1-5, April 2015

9. R. Zhang, C. Lin, K. Meng, and L. Zhu, "BEC: A reliable and efficient mechanism for cloud storage service," IEEE Intl. Conf. on Communication Technology, pp. 717-721, November 2013

10. D. Fitch, and H. Xu, "A RAID-based secure and fault-tolerant model for cloud information storage," Intl. J. of Software Engineering and Knowledge Engineering, vol. 23, pp. 627-654, April 2013

11. Q. Liu, and L. Xing, "Reliability Modeling of Cloud-RAID-6 Storage System," International Journal of Future Computer and Communication, vol. 4, no. 6, pp. 415-420, December 2015

12. F. Bausch, Cloud-RAID Concept, available at http://blog.fbausch.de/cloudraid-3-concept/, accessed in April 2017

13. T. Jin, Y. Yu, and L. Xing, "Reliability Analysis of RAID Systems using Repairable k-out-of-n Modeling Techniques," Intl. Conf. on Interface between Statistics \& Engineering, pp. 13-15, 2009

14. Q. Liu, and L. Xing, "Hierarchical Reliability Analysis of Multi-state Cloud-RAID Storage System," Proc. of Intl. Conf. on Quality, Reliability, Risk, Maintenance, \& Safety Eng., July 2015

15. A. Myers, "Complex System Reliability," Springer Series in Reliability Engineering, 2nd edition, September 23, 2010

16. T. Jin, L. Xing, and Y. Yu, "A hierarchical Markov reliability model for data storage systems with media self-recovery," International Journal of Reliability, Quality and Safety Engineering, vol. 18, pp. 25-41, November 2011

17. D. Patterson, P. Chen, G. Gibson, and R. H. Katz, "Introduction to Redundant Arrays of Inexpensive Disks (RAID)," Proceedings of Thirty-Fourth IEEE Computer Society International Conference: Intellectual Leverage, Digest of Papers, pp. 112-117, February-March 1989

18. S. V. Amari, A. Myers, and A. Rauzy, "An Efficient Algorithm to Analyze New Imperfect Fault Coverage Models," Proc. of Annual Reliability and Maintainability Symposium, pp. 420-426, January 2007

19. S. V. Amari, A. Myers, A. Rauzy, and K. Trivedi, "Imperfect coverage models: status and trends," Handbook of Performability Engineering, Editor: K. B. Misra, Springer, 2008

20. A. Myers, "k-out-of-n: G system reliability with imperfect fault coverage," IEEE Transactions on Reliability, vol. 56, no. 3, pp. 464-473, September 2007

21. A. Myers, and A. Rauzy, "Assessment of redundant systems with imperfect coverage by means of binary decision diagrams," Reliability Eng. \& System Safety, vol. 93, pp. 1025-1035, 2008

22. L. Xing, and S. V. Amari, "Binary Decision Diagrams and Extensions for System Reliability Analysis," Wiley-Scrivener, MA, ISBN: 978-1-118-54937-7, 2015

23. L. Xing, H. Wang, C. Wang, and Y. Wang, "BDD-Based Two-Party Trust Sensitivity Analysis for Social Networks," Intl. Journal of Security and Networks, vol. 7, no. 4, pp. $242-251,2012$

24. S. V. Amari, J. B. Dugan, and R.B. Misra, "A separable method for incorporating imperfect fault-coverage into combinatorial models," IEEE Trans. on Reliability, vol. 48, no. 3, pp. 267-274, 1999

25. L. Xing, and J. B. Dugan, "Analysis of Generalized Phased Mission System Reliability, Performance and Sensitivity," IEEE Transactions on Reliability, vol. 51, no. 2, pp. 199-211, 2002

Lavanya Mandava received her B.Tech. degree in electronics and computer engineering from JNTUK, India in 2011, and M.S. degree in computer engineering from the University of Massachusetts, Dartmouth in 2016. She is currently a Ph.D. student in the Department of Electrical and Computer Engineering, University of Massachusetts Dartmouth, USA.

Liudong Xing received her Ph.D. degree in electrical engineering from the University of Virginia, Charlottesville, USA in 2002. She is a Professor with the Department of Electrical and Computer Engineering, University of Massachusetts (UMass) Dartmouth, USA. Her current research interests include reliability modeling and analysis of complex systems and networks. Prof. Xing was the recipient of the Leo M. Sullivan Teacher of the Year Award in 2014, the Scholar of the Year Award in 2010, and the Outstanding Women Award in 2011 of UMass Dartmouth. She was the recipient of the Changjiang Scholar Award from the Ministry of Education of China in 2015, and IEEE Region 1 Technological Innovation (Academic) Award in 2007. She was also co-recipient of the Best (Student) Paper Award at several international conferences. She is an Associate Editor or Editorial Board member of multiple journals including Reliability Engineering \& System Safety, and International Journal of Systems Science. 